\title{
Natural history of hepatic haemangiomas: clinical and ultrasound study
}

\author{
L Gandolfi, P Leo, L Solmi, E Vitelli, G Verros, A Colecchia
}

\begin{abstract}
Hepatic haemangiomas are the most common benign tumours of the liver and commonly present as incidental findings on sonographic examination of the abdomen. Since little is known of the natural course of these tumours, we performed a clinical and sonographic follow up of 123 haemangioma patients. Our prospective study investigated clinical and sonographic findings in $\mathbf{1 5 8}$ haemangiomas for periods of 12 to 60 months. Ninety nine haemangiomas measured $<2 \mathrm{~cm}$ and had an echogenic pattern; 40 were between $2 \mathrm{~cm}$ and $5 \mathrm{~cm}$ with a mainly echogenic structure; 19 measured $>5 \mathrm{~cm}$ and showed a mixed echo pattern. At the first examination only eight patients, all with giant haemangiomas, presented symptoms which could be attributed to the tumour. During follow up only one haemangioma changed in shape and size. One patient who was symptom free at the first examination experienced right upper abdominal quadrant pain during follow up. No deterioration occurred in any of the patients with symptoms at the first examination, and all had a satisfactory quality of life. No complications arose during the follow up period. This study shows that in adults haemangiomas remain stable in size and echo patterns rarely change. Only haemangiomas $>5 \mathrm{~cm}$ may cause symptoms. Prolonged clinical and sonographic follow up of small and medium sized haemangiomas is not warranted.
\end{abstract}

Hepatic haemangiomas are the most commonly detected benign liver tumours. Before the widespread use of diagnostic imaging techniques the natural history of haemangiomas was based on necropsy findings, ${ }^{12}$ incidental surgical findings, ${ }^{3}$ or, more rarely, treatment for complications of haemangioma (intrahepatic or peritoneal bleeding). ${ }^{4}$

Gastroenterology Unit, Malpighi Hospital, Via Albertoni 15, 40138

Bologna, Italy

L Gandolfi

P Leo

L Solmi

E Vitelli

G Verros

A Colecchia

Correspondence to:

Professor Lionello Gandolfi.

Accepted for publication

3 August 1990
TABLE I Liver haemangiomas: distribution by size, sex, and age of patients at first examination, and average length of follow up

\begin{tabular}{|c|c|c|c|c|c|c|}
\hline \multirow{2}{*}{$\begin{array}{l}\text { Size } \\
(\mathrm{cm})\end{array}$} & \multirow{2}{*}{$\begin{array}{l}\text { No of } \\
\text { patients }\end{array}$} & \multirow[b]{2}{*}{ Sex } & \multicolumn{2}{|l|}{ Age (years) } & \multicolumn{2}{|c|}{$\begin{array}{l}\text { Follow up } \\
\text { (months) }\end{array}$} \\
\hline & & & $\operatorname{Mean}(S E)$ & Range & Mean & Range \\
\hline$<2$ & 26 & $\mathbf{M}$ & $\begin{array}{l}51 \cdot 6(2 \cdot 7) \\
50.4(2 \cdot 1)\end{array}$ & $\begin{array}{l}20-79 \\
34-72\end{array}$ & $\begin{array}{l}17 \cdot 5 \\
21.4\end{array}$ & $\begin{array}{l}12-38 \\
14-57\end{array}$ \\
\hline$>2-<5$ & 10 & $M$ & $55 \cdot 7(4 \cdot 0)$ & $48-69$ & $\begin{array}{l}21 \cdot 4 \\
28 \cdot 8\end{array}$ & $\begin{array}{l}14-31 \\
14-45\end{array}$ \\
\hline & 21 & $\mathrm{~F}$ & $54.4(2 \cdot 8)$ & $31-72$ & $21 \cdot 6$ & $15-39$ \\
\hline$>5$ & 5 & M & $62.8(3.9)$ & $53-76$ & $22 \cdot 8$ & $13-39$ \\
\hline & 12 & $\mathbf{F}$ & $52 \cdot 1(1 \cdot 7)$ & $51-67$ & $24 \cdot 2$ & $12-41$ \\
\hline
\end{tabular}

In view of this, little is known of the natural history of these tumours and management is still the subject of debate. As yet there is no agreement on whether giant haemangiomas should be resected to prevent rupture or further growth which could preclude subsequent surgery.

Ultrasonographic diagnosis of hepatic haemangiomas has proved an accurate technique. ${ }^{5-7}$ As it is not invasive and easily repeated, it is an ideal way of charting the course of these tumours. ${ }^{8}$ We set out to establish the natural history of haemangiomas by monitoring sonographic changes in size, shape, and echo pattern alongside clinical features.

\section{Methods}

Over a period of seven years (January 1981December 1987) haemangiomas were found in 292 out of 21280 patients who were referred to our ultrasound unit, giving a prevalence of $1 \cdot 4 \%$. Of these 292 patients, 123 were enrolled in an ultrasonographic and clinical follow up study lasting from 12 to 60 months (average 21.6 months). There were 41 men (33.4\%) and 82 women $(66.6 \%)$ aged between 20 and 79 years; mean age was $56 \cdot 1$ years for men and $52 \cdot 3$ years for women.

Lesions were found as follows: $75.6 \%$ of patients had one, $21 \cdot 2 \%$ presented with two, and only $3 \cdot 2 \%$ had three or more. Altogether, we monitored 158 haemangiomas: 99 were under $2 \mathrm{~cm}$ in size (62.6\%), 40 were between $2 \mathrm{~cm}$ and $5 \mathrm{~cm}(25 \cdot 4 \%)$, and 19 had a maximum diameter of over $5 \mathrm{~cm}(12 \cdot 0 \%)$ (Table I).

In $31 \%$ of cases haemangiomas were detected during investigation for staging or follow up of patients with neoplasia, or both. Ultrasonography was performed for suspected gall bladder or bile duct lithiasis in $22.9 \%$ of patients, dyspepsia in $21 \%$, abdominal pain in $6.5 \%$, abnormal liver function tests in $7 \cdot 3 \%$, abdominal mass in $1.6 \%$, and other causes in $10.5 \%$.

In the largest group of lesions measuring under $2 \mathrm{~cm}$ the final diagnosis was established on the basis of sonographic features, clinical observation, negative biochemical results (in particular, aspartate and alanine transaminase, alkaline phosphatase, and $\gamma$ glutamyltranspeptidase activities and concentrations of carcinoembryonic antigen and $\alpha$ fetoprotein), and above all the findings of at least six months' clinical and sonographic follow up, which is a valid diagnostic criterion. ${ }^{79}$

The final diagnosis was made for haeman- 


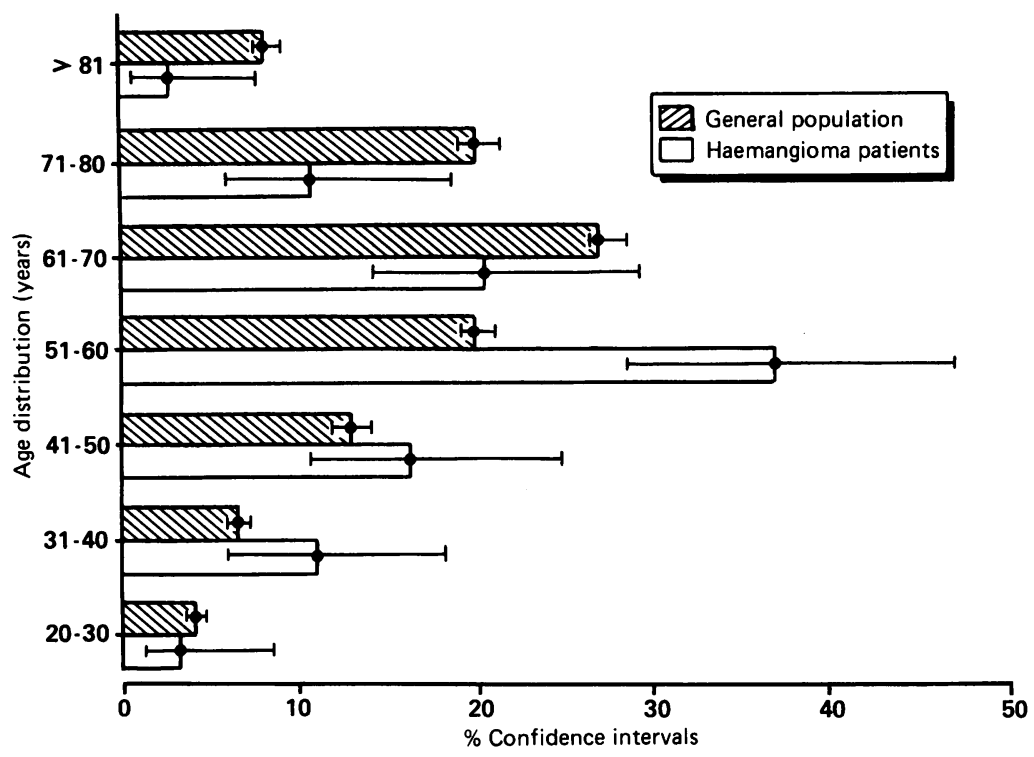

Figure 1: Age distribution of haemangioma patients (open columns) compared with that of patients referred for ultrasound examination (hatched columns). $95 \%$ confidence intervals shown.

giomas between $2 \mathrm{~cm}$ and $5 \mathrm{~cm}$ using imaging techniques in 34 lesions (computed tomography, ${ }^{99 m}$ technetium-red blood cell radionuclide scans, laparoscopy). The remaining seven haemangiomas were diagnosed according to the above clinical, biochemical, and sonographic criteria; none of these patients had a past history of malignancy.

In all 17 patients with giant haemangiomas the final diagnosis was made by computed tomography (nine cases), ${ }^{99 \mathrm{~m}} \mathrm{Tc}$-red blood cell radionuclide scans (six cases), laparoscopy (four cases), and angiography (four cases). Some patients were subjected to more than one imaging technique.

Ultrasonography was performed with an SSD Aloka 256 real time scanner using a $3.5 \mathrm{MHz}$ linear transducer; images were recorded by Polaroid. At least three transverse, longitudinal, and anteroposterior measurements were taken of each lesion. When none of the three diameters coincided with the maximum diameter of the haemangioma a section containing the maximum diameter was measured. Changes in size within an upper or lower limit of $2 \mathrm{~mm}$ were not considered important owing to the measurement limitations of the equipment.

Clinical and sonographic follow up was performed in all patients at three and six months in the first year and annually thereafter.

\section{Results}

The male:female ratio of the 123 haemangioma patients in the follow up study was $1: 2$. This ratio was not significantly different from that found in the 292 patients found to have haemangioma (95\% confidence interval). Average age was

TABLE II Correlation between size and echo pattern in 158 haemangiomas at first examination

\begin{tabular}{lllr}
\hline & \multicolumn{3}{l}{ Size $(\mathrm{cm})$} \\
\cline { 2 - 4 } Echo pattern & $<2$ & $>2-<5$ & $>5$ \\
\hline Hyperechoic & 98 & 33 & 3 \\
Hypoechoic & 0 & 2 & 1 \\
Mixed & 1 & 5 & 15 \\
Total & 99 & 40 & 19 \\
\hline
\end{tabular}

similar for men and women in the two groups with lesions of $\leqslant 2 \mathrm{~cm}$ and between $2 \mathrm{~cm}$ and $5 \mathrm{~cm}$, while there was a difference of a decade in the group with haemangiomas over $5 \mathrm{~cm}$. Results are summarised in Table I. The age distribution of the patients with a haemangioma does not reflect the age distribution of patients referred for ultrasound examination (Fig 1).

\section{ULTRASONOGRAPHIC FOLLOW UP}

The sonographic appearance of the haemangiomas at the first observation was hyperechoic in $84.8 \%$ of cases and hypoechoic in only $1.8 \%$. The lesions presented a mixed echo pattern in $13.4 \%$, with hyperechoic areas alternating with hypoechoic or anechoic areas. All haemangiomas under $2 \mathrm{~cm}$ had an echogenic pattern. Those measuring between $2 \mathrm{~cm}$ and $5 \mathrm{~cm}$ were mainly hyperechoic, but a mixed echo pattern was seen in $12 \%$ of cases. Lesions over $5 \mathrm{~cm}$ presented mainly mixed echo patterns ( $78 \%)$ as reported in Table II. During the follow up period there were no appreciable changes in the size and sonographic features of haemangiomas under $2 \mathrm{~cm}$ and those between $2 \mathrm{~cm}$ and $5 \mathrm{~cm}$. Most of these lesions presented a homogeneous hyperechoic pattern, which simplified the comparison of sonograms over time.

In the group of giant haemangiomas (diameter over $5 \mathrm{~cm}$ ) the sonographic features changed in one man during the third year of follow up. The maximum diameter of his lesion had increased by $1.5 \mathrm{~cm}$ and wide echo free areas were present in the middle of the haemangioma (Fig 2). This enlargement was confirmed by computed tomography with bolus infusion of contrast medium compared with the previous scan three years earlier. In the other 16 patients the sonograms were unchanged.

\section{CLINICAL FOLLOW UP}

All the haemangiomas under $2 \mathrm{~cm}$ or between $2 \mathrm{~cm}$ and $5 \mathrm{~cm}$ were found incidentally during ultrasound examination, usually during screening for liver metastases in cancer patients or for suspected biliary disease. During follow up none of these patients presented symptoms which could be correlated with the haemangioma.

Eight of the 17 patients with hepatic haemangiomas over $5 \mathrm{~cm}$ were symptomatic at the first examination: seven reported discomfort in the right upper abdominal quadrant and feelings of fullness (but three of these also had gall bladder lithiasis); one patient presented with an episode of abdominal pain with fever, slight jaundice, and choluria (the lesion was located in segment IV). The other nine patients were asymptomatic at the first examination, although five had clinically evident hepatomegaly and in two an abdominal mass was palpable. The other three patients screened presented no symptoms or clinical signs at the first examination.

During follow up, eight of the nine asymptomatic patients remained symptom free. One patient experienced an acute episode of pain in the right upper abdominal quadrant during physical exercise, which lasted several hours and was accompanied by raised activity of hepatic 

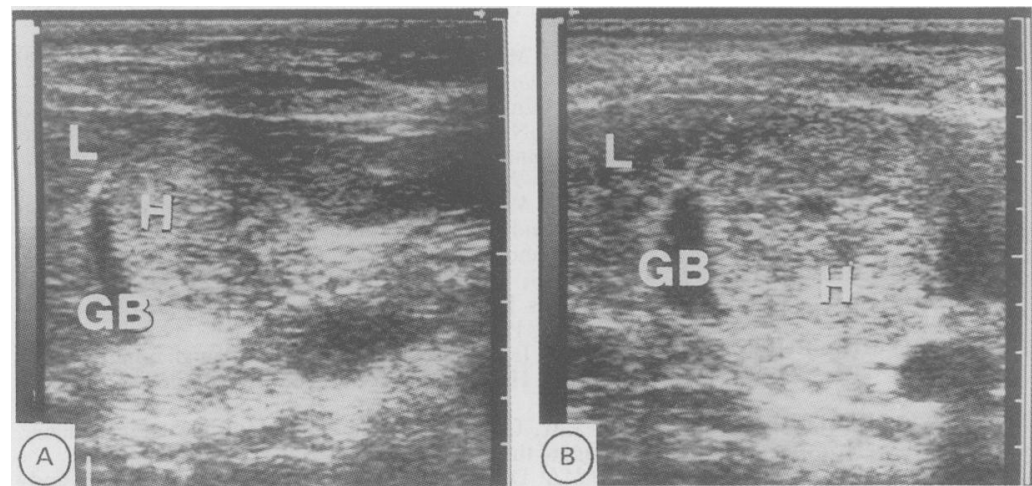

Figure 2: Transverse scan. (A) Haemangioma $(H)$ in liver segment $I V$ at first examination; $(B) 36$ months later the tumour had enlarged and an echo free central area is evident. $L=$ liver, $G B=$ gall bladder.

enzymes of cholestasis and cytolysis. Ultrasonography disclosed no changes in size or echo pattern and biochemical parameters soon normalised.
Symptoms subsided in six of the eight patients with giant lesions who were symptomatic at the first examination. The patient with abdominal pain and choluria has not had recurrent episodes during a three year follow up even though his haemangioma had increased in diameter. Symptoms remained unchanged in two patients but their quality of life has been acceptable. During follow up none of the patients with a giant haemangioma had major complications such as bleeding, rupture, fever, or thrombocytopenia.

\section{Discussion}

Haemangiomas are the most common benign liver tumours, ${ }^{10}$ with a prevalence varying from $1 \cdot 4 \%$ to $2 \cdot 3 \%$ in necropsy, surgical, ${ }^{12}$ or, more recently, ultrasonographic series. ${ }^{12}$ In our series the prevalence was found to be $1 \cdot 4 \%$.

The diagnostic problems related to the frequent occurrence of this tumour have been largely resolved by state of the art imaging techniques (computed tomography and ultrasonography). ${ }^{7913}$ Above all, ultrasonography has provided a great deal of information on the natural course of these lesions of which little has been known because of the lack of published reports. ${ }^{814}$

The male:female ratio of the patients in our clinical and ultrasonographic follow up was $1: 2$, in agreement with other series ${ }^{1314}$ but lower than reported by other workers who noted a female prevalence of up to $5: 1 .^{315}$

Incidental detection of haemangiomas is most common in the third, fourth, and fifth decades of life. ${ }^{16}$ In our series the average age was similar for men ( 56.7 years) and women $(52.3$ years). Considering only those patients with so called giant haemangiomas (diameter over $5 \mathrm{~cm}$ ), the average age at detection was a decade lower in women than in men, as reported by Trastek et al. ${ }^{1+}$ This finding is not related to contraceptive use, as none of the women in our series had a history of oral contraceptive use.

During our follow up study the size and sonographic features of small and medium sized haemangiomas remained unchanged. In the group with giant lesions over $5 \mathrm{~cm}$ only one tumour increased in size with a changed echo pattern, but this finding was incidental and not accompanied by symptoms. Haemangioma enlargement has been reported in other series, ${ }^{814}$ and there is general agreement that the lesions increase in size after bleeding or intratumoral thrombosis or because of ectasia of the vascular channels rather than because of new growth. ${ }^{211} 17$

The role of female hormones in the development and growth of hepatic haemangiomas is under discussion. ${ }^{18}$ These lesions arise mainly in women and rapid tumour growth has been reported in a 57 year old woman after exogenous oestrogen administration ${ }^{19}$ and during pregnancy. ${ }^{2021}$ Two cases of haemangiomas complicated by intraperitoneal bleeding have also been described in pregnant women. ${ }^{4}$ Haemangiomas remained unchanged in the group of women who experienced menopause during the follow up period, and our only case of tumour enlargement was in a young man. None of the haemangiomas decreased in size even though reduction has been described $^{814}$ and may be due to fibrous replacement. ${ }^{22}$

Clinical symptoms are present in only a small proportion of haemangioma patients ${ }^{14} 182023$ and only those with giant lesions ${ }^{1124}$; symptoms include abdominal swelling, abdominal mass, hepatomegaly, sudden upper abdominal quadrant pain, and symptoms arising from compression of surrounding organs. In our series $6.5 \%$ of patients were symptomatic at the first examination with symptoms directly related to the tumour, and none experienced a worsening of symptoms during follow up.

As regards patients who were symptom free at the first examination, only one had symptoms during follow up, consisting of sudden abdominal pain in the right upper quadrant. His accompanying symptoms (mild jaundice, increased alkaline phasphatase and $\gamma$ glutamyltransferase) and the position of the haemangioma (segments IV and V) suggest that the pain he experienced could have been due to transient bile duct compression. Another patient presented at the first examination with pain and mild jaundice mimicking biliary colic, and similar cases have been reported in other series. ${ }^{16}$ Although when present symptoms depend on the size of the haemangioma, according to Ishak and Rabin, ${ }^{18}$ we believe that tumours located in segments IV and $\mathrm{V}$ are likely to cause discomfort. As the gall bladder and bile ducts are adjacent to this area, symptoms may occur even when haemangiomas are not particularly large.

Older reports have noted a high percentage of complications in adult haemangioma patients varying from $4 \cdot 5 \%$ to $19 \cdot 7 \% .^{18}$ The major risk is rupture with intraperitoneal haemorrhage, which is fatal in $75 \%$ of cases ${ }^{4}$ but fortunately is rare in tumours under $10 \mathrm{~cm} .{ }^{9}$ There are only anecdotal reports of life threatening complications in more recent series, ${ }^{25-27}$ indicating that risks are small compared with the high incidence of haemangiomas. During our follow up none of the patients with giant haemangiomas had any complications, although the limited length of follow up should be taken into account. There is no evidence that haemangiomas become malignant, and none of the cases monitored in our series became malignant.

The results of our follow up and those in 
recent studies reporting few complications ${ }^{814}$ raise the question of whether the risks of surgically resecting giant asymptomatic haemangiomas outweigh the risks of haemorrhage or enlargement. In fact, surgical management carries some degree of risk..$^{14202728}$ So far alternative therapeutic approaches, such as hepatic artery ligation or embolisation, have failed to shrink haemangiomas or improve symptoms and they carry the risk of ischaemia and infection. 142629

Our study shows that prolonged ultrasound follow up of small and medium sized haemangiomas is not warranted. Short term follow up (6-12 months) is useful when the final diagnosis has not been established by other techniques in order to differentiate the haemangioma from malignant lesions, especially secondary tumours in cancer patients.

1 Adami JG. The principles of pathology. Philadelphia: Lea and Febiger, 1910

2 Ochsner JL, Halpert B. Cavernous hemangioma of the liver. Surgery 1958; 43: 577-82.

3 Shumaker HB Jr. Hemangioma of the liver: discussion of symptomatology and report of patient treated by operation. symptomatology and report

4 Sewell JH, Weiss K. Spontaneous rupture of hemangioma of the liver: a review of the literature and presentation of illustrative case. Arch Surg 1961; 83: 729-33.

5 McArdle CR. Ultrasonic appearance of a hepatic hemangioma. $\Im$ Clin Ultrasound 1978; 6: 124.

6 Freeny PC, Vimont TR, Barnett DC. Cavernous hemangiomas of the liver: ultrasonography, arteriography and computed tomography. Radiology 1979; 132: 143-8.

7 Gandolfi L Solmi L, Bolondi L, Rossi A Casanova P, Leo P. The value of ultrasonography in the diagnosis of hepatic haemangiomas. Eur $\mathcal{F}$ Radiol 1983; 3: 222-6.

8 Gibney RG, Hendin AP, Cooperberg PL. Sonographically detected, Hendin AP, Cooperberg PL. Sonographically detected hepatic hemangiom

9 Taylor CR, Taylor KJW. An incidental hemangioma of the liver: the dilemma of patient management. $\mathcal{F}$ Clin Gastroenterol 1981; 3: 93-7.
10 Feldman M. Hemangioma of the liver. Am $\mathcal{F}$ Clin Pathol 1958; 29: $160-2$

11 Grieco MB, Miscall BG. Giant hemangiomas of the liver. Surg Gynecol Obstet 1978; 147: 783-7.

12 Itai $Y$, Ohtomo K, Furui S, Yamauchi T, Minani M, Yaschiro $\mathrm{N}$. Non invasive diagnosis of small cavernous hemangioma of the liver: advantage of MRI. AYR 1985; 145: 1195-9.

13 Freeny PC, Marks WM. Patterns of contrast enhancement of benign and malignant hepatic neoplasms during bolus benign and malignant hepatic neoplasms during

14 Trastek VF, van Heerden JA, Sheedy PF, Adson MA Cavernous hemangiomas of the liver: resect or observe? Am ₹ Surg 1983; 145: 49-53.

15 Henson SW Jr, Gray HK, Dockerty MB. Benign tumors of the liver. II. Hemangiomas. Surg Gynecol Obstet 1956; 103 . 327-31.

16 Levitt LM, Coleman M, Yarvis J. Multiple large hemangiomas of the liver. $N$ Engl $\mathcal{F}$ Med 1955; 252: 854 .

17 Adson MA. Diagnosis and surgical treatment of primary and secondary solid hepatic tumors in the adult. Surg Clin North Am 1981; 61: 181-96.

18 Ishak KG, Rabin L. Benign tumor of the liver. Med Clin North Am 1975; 59: 995-1013.

19 Morley JE, Myers JB, Sacks FS, Kalk F, Epstein EE, Lannon J. Enlargement of cavernous hemangioma associated with exogenous administration of oestrogens. S Afr Med f 1974 48: 695-7.

20 Borman PC, Terblanche J, Blumgart RL, Harries Jones EP Pickard H, Kalvaria I. Giant hepatic hemangiomas: diagnostic and therapeutic dilemmas. Surgery 1987; 101: 445-9.

21 Issa P. Cavernous haemangioma of the liver: the role of radiotherapy. Br $\mathcal{F}$ Radiol 1968; 41: 26-32.

22 Bree RL, Schwab RE, Neiman HL. Solitary echogenic spot in the liver: is it diagnostic of a hemangioma? AfR 1983;140. the liv.

23 Scatarige JC, Kenny JM, Fishman EK, Herlong FH, Siegel man SS. CT of giant cavernous hemangioma. $A \mathcal{F R} 1987$ 149: $83-5$

24 Adam YG, Huvos AG, Fortner JC. Giant hemangiomas of the liver. Ann Surg 1970; 172: 239-45.

25 Kato M, Sugawara I, Okada A, et al. Hemangioma of the liver: diagnosis with combined use of laparoscopy and hepatic arteriography. Am $\mathcal{F}$ Surg 1975; 129: 698-704.

26 Stayman JW Jr, Polsky HS, Blaum L. Case report: ruptured cavernous hemangioma of the liver. $\mathrm{Pa} \mathrm{Med} \mathrm{1976;79:}$ 62-3.

27 Starzl TE, Koep LJ, Weil R III, et al. Excisional treatment of cavernous hemangioma of the liver. Ann Surg 1980; 192: $25-7$

28 Kawadara Y, Mizumolo R. Surgical treatment of giant hemangioma of the liver. Am $\mathcal{F}$ Surg 1984; 148: 287-91.

29 Martin B, Roche A, Radice L, Aguilar K, Kraiem C. Does arterial embolization have a role in the treatment of cavernous hemangioma of the liver? Presse Med 1986; 15: 1073-6. 